Original Article

\title{
Choice of Aortic Valve Prosthesis in a Rapidly Aging and Long-Living Society
}

\author{
Yoshimasa Sakamoto, MD, PhD, Michio Yoshitake, MD, PhD, Yoko Matsumura, MD, PhD, \\ Hitomi Naruse, MD, Ko Bando, MD, PhD, and Kazuhiro Hashimoto, MD, PhD
}

\begin{abstract}
Purpose: The aim of this study was to evaluate the long-term results of aortic valve replacement (AVR) with mechanical $(\mathrm{M})$ and bioprosthetic $(\mathrm{B})$ valves as recommended by the Japanese guidelines.

Methods: From April 1995 to March 2014, 366 adult patients underwent AVR. Of these, $127(35 \%)$ patients received $M$ and 239 patients $(65 \%)$ received $B$ valves. A retrospective analysis of the entire and the selected 124 patients aged 60 to 70 years was carried out. Results: In patients aged 60 to 70 years, the 15 -year survival and freedom from reoperation were $88 \% \pm 7 \%$ and $100 \%$ for the $M$ group and $34 \% \pm 25 \%(p<0.001)$ and $73 \% \pm 14 \%$ $(p=0.059)$ for the B group, respectively. Among propensity score matching of the subgroup, there was no significant difference in survival and freedom from reoperation. The rate of thromboembolism was higher in the $M(M: 0.58 \%$ vs B: $0.35 \%$ patient per year, $p<0.001$ ) and the rate of hemorrhage was higher in the M group (M: $0.34 \%$ vs B: $0.12 \%$ patient per year, $p<0.001$ ).

Conclusion: The current strategy of aortic valve choice based on the Japanese guidelines has provided excellent long-term results so far.
\end{abstract}

Keywords: valve choice, aortic valve replacement, heart valve prosthesis, long-term outcomes

\section{Introduction}

The current Japanese guidelines recommend a bioprosthetic (B) valve for aortic valve replacement (AVR) in patients aged more than 65 years. The staff surgeons in our facility have chosen the aortic prosthesis for each patient according to the current guidelines after discussing with the patients and their families and taking into account several factors such as age, comorbidities, and surgical/ reoperation risk. However, the use of bioprosthetic valves in the aortic position has markedly increased in recent

Department of Cardiac Surgery, Jikei University School of Medicine, Minato-Ku, Tokyo, Japan

Received: April 19, 2016; Accepted: August 12, 2016

Corresponding author: Yoshimasa Sakamoto, MD, PhD. Department of Cardiac Surgery, Jikei University School of Medicine, 3-25-8 Nishishinbashi, Minato-Ku, Tokyo 105-8461, Japan Email: ysakamoto@jikei.ac.jp

(C)2016 The Editorial Committee of Annals of Thoracic and Cardiovascular Surgery. All rights reserved. years as the number of elderly patients in Japan has increased. The details of valve durability and anticoagulation therapy are sometimes beyond patient's ability to fully grasp, and many patients leave the choice of valve type to their surgeon's recommendation, based on his experience and preferences. Sometimes, clinicians find it hard to choose which type of valve to use for patients aged 60 to 70 years. This is because recent increases in Japanese longevity make it likely that the patient will outlive the useful life of a B valve and might require reoperation as an octogenarian, particularly if the patient is a female (females tend to outlive males in Japan, as elsewhere). In this study, we analyzed the clinical outcomes after AVR in Japan's rapidly aging society, focusing on the valve choice in the age group of 60 to 70 years through propensity score matching.

\section{Patients and Methods}

The university's research ethical committee approved this study and individual patient consent was waived. 
Table 1 Preoperative patient characteristics

\begin{tabular}{lccc}
\hline Variables & $\mathrm{M}(\mathrm{n}=127)$ & $\mathrm{B}(\mathrm{n}=239)$ & $p$ Value \\
\hline Age $(\mathrm{y})$ & $55.5 \pm 13.6$ & $72.4 \pm 6.8$ & $<0.001$ \\
Male $(\%)$ & $96(75.6)$ & $133(55.6)$ & $<0.001$ \\
Body surface area $\left(\mathrm{m}^{2}\right)$ & $1.66 \pm 0.19$ & $1.54 \pm 0.18$ & 0.999 \\
Mean follow-up $(\mathrm{y})$ & $7.3 \pm 5.9(1-19)$ & $3.8 \pm 3.7(1-16)$ & $<0.001$ \\
AS & $19(15 \%)$ & $99(41 \%)$ & $<0.001$ \\
ASR & $30(24 \%)$ & $81(34 \%)$ & 0.196 \\
AR & $73(57 \%)$ & $57(24 \%)$ & $<0.001$ \\
Bicuspid & $17(13 \%)$ & $21(9 \%)$ & 0.219 \\
NYHA functional class III or IV (\%) & $6(4.7)$ & $19(7.9)$ & 0.238 \\
Diabetes $(\%)$ & $11(8.7)$ & $32(13.4)$ & 0.181 \\
Valve size (mm ) & $22.9 \pm 3.3$ & $20.9 \pm 2.0$ & $<0.0001$ \\
Endocarditis $(\%)$ & $12(9.4)$ & $10(4.2)$ & 0.044 \\
CKD (creatinine $>2$ mg/dL) $(\%)$ & $9(7.1)$ & $32(13.4)$ & 0.069 \\
Peripheral vascular disease $(\%)$ & $9(7.1)$ & $24(10.0)$ & 0.348 \\
Low preoperative EF $(<40 \%)(\%)$ & $5(3.9)$ & $7(2.9)$ & 0.598 \\
CABG $(\%)$ & $13(10.2)$ & $57(23.8)$ & 0.001 \\
\hline
\end{tabular}

NYHA: New York Heart Association; EF: ejection fraction; CKD: chronic kidney disease; CABG: coronary artery bypass grafting; AS: aortic stenosis; ASR: aortic stenosis and regurgitation; AR: aortic regurgitation

\section{Patient population}

Between April 1995 and March 2014, 366 adult patients underwent single AVR or combined AVR/ coronary artery bypass grafting $(\mathrm{CABG})(\mathrm{n}=61)$ at the Jikei University Hospital. In all, 127 (35\%) patients received $\mathrm{M}$ valves, and $239(65 \%)$ patients received $\mathrm{B}$ valves. The characteristics of the patients are summarized in Table 1. Follow-up was 94\% complete, with a mean duration of $5.4 \pm 4.5$ years (range: $1-17.7$ ).

\section{Choice of prosthesis}

The main criterion for choosing a B valve in the aortic position was age older than 65 years and the fundamental principle of valve selection was based on the current Japanese guidelines (http://www.j-circ.or.jp/guideline/ pdf/JCS2012_ookita_h.pdf). The types and numbers of $B$ valves used were Carpentier-Edwards Perimount (100); Magna (32) and Magna EASE (30; Edwards Lifesciences, Irvine, CA, USA); Medtronic Mosaic (28) and Ultra (22; Medtronic, Minneapolis, MN, USA); and St. Jude Medical (SJM) Trifecta (25) and SJM Epic (2; St. Jude Medical, St. Paul, Minnesota, MN, USA). The types of M valves inserted were SJM Standard (74) and SJM Regent (25; St. Jude Medical); ATS (27; ATS Medical, Minneapolis, MN, USA); and CarboMedics (1; Sorin SpA, Milan, Italy). These valves were chosen because they were commonly implanted during the study period at our institute.

\section{Surgical procedure and management of anticoagulation}

A midline sternotomy was performed in all the patients. After resection of the native aortic valve cusps, the valve size was measured with the sizer provided by the prosthesis manufacturer. The $\mathrm{B}$ valves were implanted in the supra-annular position with pledgeted 2-0 polyester sutures with a non-everting mattress suture technique. The $\mathrm{M}$ valves were implanted in the intraannular position (with the exception of small valve sizes) using an everting mattress suture technique. In patients with a $\mathrm{B}$ valve, the target international normalized ratio (INR) was maintained between 1.5 and 2.5 for 3 months. Thereafter, patients received only aspirin, if not otherwise indicated. In patients with a mechanical prosthesis, INR has been maintained lifelong between 2.0 and 2.5.

\section{Statistical analysis}

Valve-related morbidity and mortality were reported according to definitions given in the Guidelines of the European Association for Cardio-thoracic Surgery. ${ }^{1}{ }^{1}$ Data are expressed as the mean \pm standard deviation (SD) and were compared using the paired Student's $t$ test. A probability value of less than 0.05 was considered significant. Cumulative survival and freedom from reoperation were analyzed using the Kaplan-Meier method, and curves were compared with the log-rank test. In addition, propensity score analysis was performed through matching 


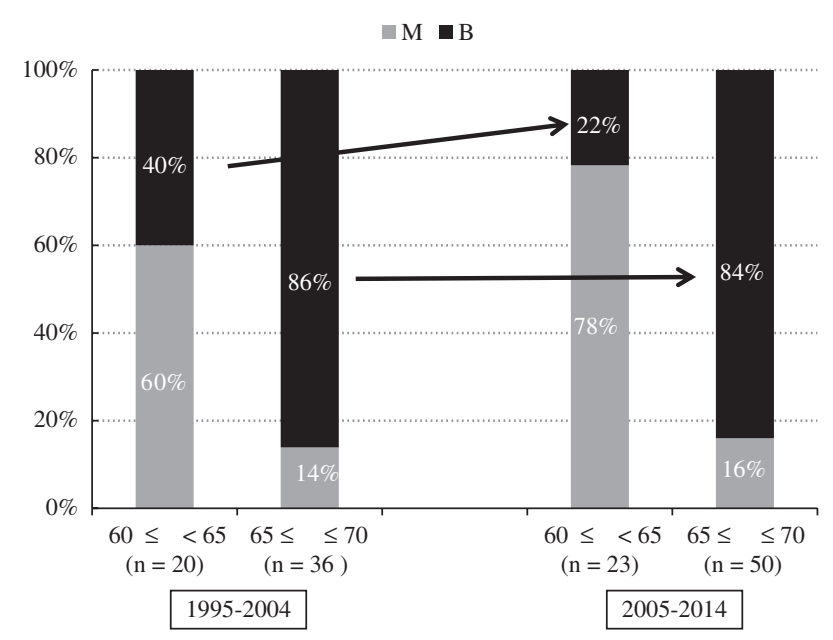

Fig. 1 Recent changes of aortic valve choices in patients aged between 60 and 70 years.

between the two groups. The 14 known preoperative potential confounders were included in the logistic regression model, and the propensity scores were calculated for each patient. Using the propensity score, the patients with $M$ valves were matched with the patients with $\mathrm{B}$ valves by a 5 to 1 digit matching algorithm using the nearest available one-to-one pair matching method. Statistical calculations were performed using a computerized statistical program (IBM SPSS Statistics 22).

\section{Results}

\section{Recent changes in patient's age and valve choice}

The mean age at surgery increased from $59 \pm 13$ years during the first half of the study period to $68 \pm 12$ ( $p<0.001$ ) years during the second half of the study period. Likewise, the use of the $\mathrm{B}$ valves increased from $55 \%$ to $71 \%$ during these periods. The valve size became smaller in both the M and B groups (M: $25.0 \pm 1.5$ to 21.6 $\pm 3.5 \mathrm{~mm}, \mathrm{~B}: 21.7 \pm 2.3$ to $20.6 \pm 1.7 \mathrm{~mm}$ ) during these periods. Although the total number of patients who received $\mathrm{B}$ valves increased from $55 \%$ to $71 \%$ during these periods, the proportion of patients aged 60 to 70 years who received B valves decreased from $70 \%$ to $64 \%$. Moreover, the number of patients aged 60 to 65 years who received B valves decreased from $40 \%$ to $22 \%$ (Fig. 1).

\section{Perioperative histories and late outcomes}

During the perioperative period, four patients died in the $\mathrm{M}$ group and eight patients died in the B group (3.1\% vs $3.3 \%, p=0.92$ ) (Table 2). The 5-, 10-, and 15-year overall actuarial survival was $88 \% \pm 3 \%, 85 \% \pm 4 \%$, and
Table 2 Hospital and Late death

\begin{tabular}{lcc}
\hline Hospital death & M (3.9\%) & B (3.3\%) \\
Cause & 1 & 3 \\
LOS & 1 & 1 \\
Pneumonia & 1 & 1 \\
Cerebrovascular accident & 1 & 1 \\
PVE & 1 & 0 \\
DIC & 0 & 1 \\
Lung abscess & 0 & 1 \\
GI bleeding & M (6.2\%) & B (4.2\%) \\
Late death & 2 & 4 \\
Cause & 1 & 1 \\
Cancer & 1 & 1 \\
Sudden death & 0 & 3 \\
PVE & 2 & 0 \\
Cerebrovascular accident & 1 & 1 \\
AMI & 1 & 0 \\
Rupture of TAA & & \\
Pneumonia & & \\
\hline
\end{tabular}

LOS: low output syndrome; PVE: prosthetic valve endocarditis; DIC: disseminated intravascular coagulation; GI: gastrointestine; AMI: acute myocardial infarction;

TAA: thoracic aortic aneurysm

$85 \% \pm 4 \%$ for the M group and $86 \% \pm 3 \%, 55 \% \pm 6 \%$, and $43 \% \pm 7 \%$ for the B group, respectively $(p=0.220)$. The 15 -year overall freedom from reoperation was $98 \%$ $\pm 2 \%$ for the M group and $89 \% \pm 5 \%$ for the B group $(p=0.015)$. Only one patient $(0.8 \%)$ underwent reoperation in the $\mathrm{M}$ group because of prosthetic valve endocarditis. In the B group, eight patients (3.3\%) underwent reoperation. Of these, four patients were diagnosed as structural valve deterioration (SVD) and the others were prosthetic valve endocarditis.

\section{Outcomes in patients aged 60 to 70 years after propensity score matching}

There were 124 (34\%) patients aged 60 to 70 years. In total, 41 patients (33\%) received $\mathrm{M}$ valves and 83 patients $(67 \%)$ received B valves. We selected 14 variables to eliminate the differences between the $\mathrm{M}$ and the $\mathrm{B}$ groups, and propensity score matching was performed. After constituting the propensity-matched $\mathrm{M}$ and $\mathrm{B}$ groups, our final study population generated 28 matched pairs (Table 3 ). Using one-to-one matching, there was no significant difference in actuarial survival and freedom from reoperation between the matched M and B groups (Figs. 2A and 2B).

\section{Thromboembolism and hemorrhage}

There were significant differences in the linearized rate ( $\%$ patient per year) of thromboembolism and 
Table 3 Preoperative patient characteristics aged 60 to 70 years before and after propensity matching

\begin{tabular}{|c|c|c|c|c|c|c|}
\hline & \multicolumn{3}{|c|}{ Unadjusted } & \multicolumn{3}{|c|}{ Adjusted } \\
\hline & $M(n=41)$ & $\mathrm{B}(\mathrm{n}=83)$ & $p$ Value & $M(n=28)$ & $\mathrm{B}(\mathrm{n}=28)$ & $p$ Value \\
\hline Age (y) & $63.7 \pm 2.9$ & $67.3 \pm 2.5$ & $<0.001$ & $64.3 \pm 2.8$ & $65.3 \pm 2.6$ & 0.112 \\
\hline Male $(\%)$ & $26(63.4)$ & $44(53.0)$ & 0.275 & $16(57.1)$ & $12(42.9)$ & 0.285 \\
\hline Body surface area $\left(\mathrm{m}^{2}\right)$ & $1.65 \pm 0.21$ & $1.57 \pm 0.18$ & 0.021 & $1.58 \pm 0.16$ & $1.49 \pm 0.21$ & 0.032 \\
\hline Mean follow-up (y) & $5.9 \pm 4.8$ & $5.2 \pm 4.3$ & 0.572 & $7.0 \pm 5.6$ & $7.8 \pm 5.7$ & 0.596 \\
\hline NYHA functional class & $1.7 \pm 0.5$ & $1.5 \pm 0.7$ & 0.024 & $1.7 \pm 0.5$ & $1.3 \pm 0.6$ & 0.003 \\
\hline NYHA functional class III or IV (\%) & $1(2.4)$ & $10(12.0)$ & 0.078 & 0 & $1(3.6)$ & 0.313 \\
\hline Diabetes $(\%)$ & $5(12.1)$ & $7(8.4)$ & $<0.001$ & $2(7.1)$ & $3(10.7)$ & 0.639 \\
\hline Valve size (mm) & $22.5 \pm 2.8$ & $21.4 \pm 2.3$ & 0.049 & $22.5 \pm 2.8$ & $21.4 \pm 2.3$ & 0.049 \\
\hline Endocarditis (\%) & $1(2.4)$ & $5(6.0)$ & 0.386 & 0 & $2(7.1)$ & 0.15 \\
\hline CKD $($ creatinine $>2 \mathrm{mg} / \mathrm{dL})(\%)$ & $5(13.1)$ & $3(3.6)$ & 0.068 & $2(7.1)$ & $4(12.1)$ & 0.388 \\
\hline Peripheral vascular disease $(\%)$ & $4(9.8)$ & $10(12.0)$ & 0.707 & $2(7.1)$ & $2(7.1)$ & 1 \\
\hline Low preoperative $\mathrm{EF}(<40 \%)(\%)$ & $2(4.9)$ & $10(12.0)$ & 0.207 & $2(7.1)$ & $1(3.6)$ & 0.553 \\
\hline $\operatorname{CABG}(\%)$ & $7(17.0)$ & $9(10.8)$ & 0.334 & $3(10.7)$ & $3(10.7)$ & 1 \\
\hline Previous cardiac surgery $(\%)$ & $3(7.3)$ & $5(6.0)$ & 0.785 & $3(10.7)$ & $1(3.6)$ & 0.299 \\
\hline
\end{tabular}

NYHA: New York Heart Association; CKD: chronic kidney disease; EF: ejection fraction; CABG: coronary artery bypass grafting
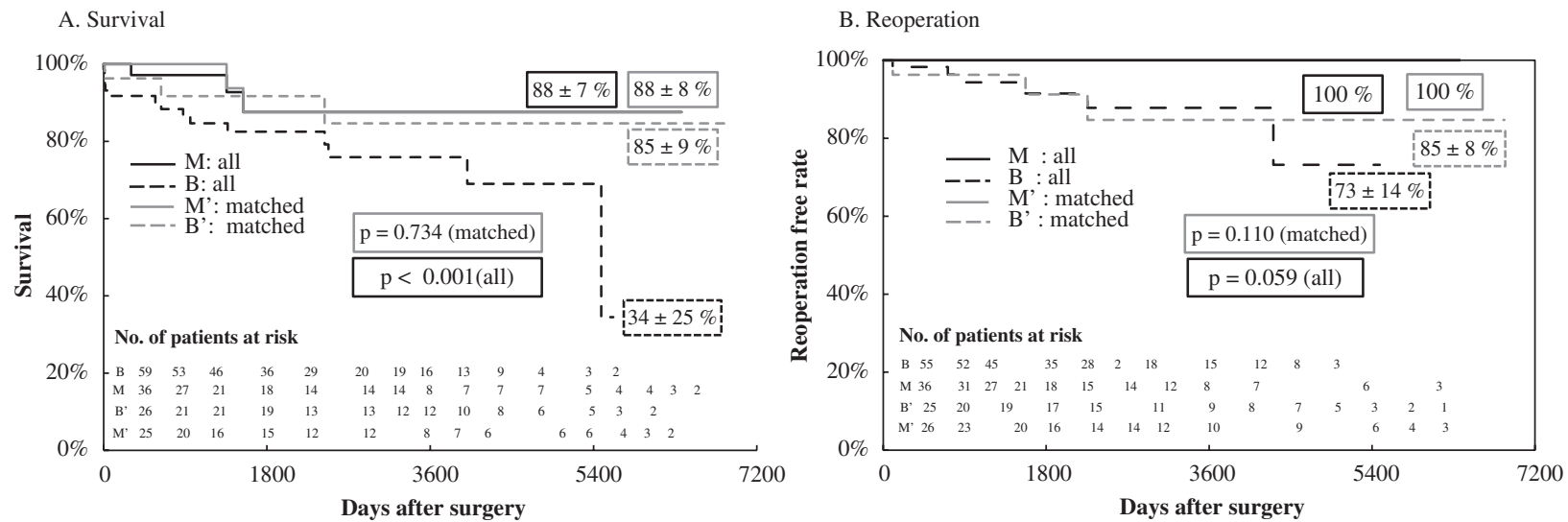

Fig. 2 Kaplan-Meier curves of survival and reoperation in patients aged 60 to 70 years before and after propensity score matching.

hemorrhage between $\mathrm{M}$ and $\mathrm{B}$ groups. The rate of thromboembolism was higher in the $\mathrm{M}$ group (M: $0.58 \%$ vs $\mathrm{B}: 0.35 \%$ patient per year, $p<0.001)$ and the rate of hemorrhage was higher in the M group (M: $0.34 \%$ vs B: $0.12 \%$ patient per year, $p<0.001$ ).

\section{Discussion}

There have been only two large randomized studies comparing the outcomes of $\mathrm{M}$ and $\mathrm{B}$ valves, and both were performed in the late 1970s: the Edinburgh Heart Valve Trial $^{2}$ and the Veterans Affairs (VA) Trial. ${ }^{3)}$ Both randomized studies had roughly similar findings, namely, higher bleeding and lower mortality/reoperation with the use of $\mathrm{M}$ valves. These data are quite consistent with the current reports in summary. In 2009, a randomized study conducted by Stassano et al. in patients aged
55 to 70 years (which they regarded as the threshold age range) yielded almost the same results as the previous two randomized studies. ${ }^{4)}$ The term "threshold age" is the age at which an implanted bioprostheses will likely outlive the patient. ${ }^{5)}$

In this study, the use of B valves markedly increased from 55\% between 1995 and 2004 to $71 \%$ between 2005 and 2013. This was primarily due to the rapid increase in the number of elderly patients. In particular, the number of patients over 70 years of age increased from $30 \%$ between 1995 and 2004 to 60\% between 2005 and 2013. In 2013, in Japan, bioprostheses accounted for $78 \%$ of all valves implanted in the aortic position, whereas in 2003 they accounted for only 38\%; in the mitral position in 2013, bioprostheses accounted for $42 \%$ of all valves implanted, whereas only $23 \%$ in 2003. ${ }^{6}$ Japan has the world's fastest aging population. Japan is predicted to 
change from an aging society (the proportion of the population of those 65 years of age or older at $7 \%$ or higher) to an aged society (the proportion of the population of those 65 years of age or older at $14 \%$ or higher) in 24 years, whereas in France, Sweden, and the UK, this change is predicted to occur in 115,85 , and 47 years, respectively ("World Population Prospects: The 2008 Revision Population Database," United Nations). Bioprostheses are widely believed to be inferior to mechanical valves in terms of durability and they are associated with a significantly higher rate of reoperation than mechanical prostheses. Recently, however, there have been several papers that recommended B valves for patients younger than 65 years because of its improved durability. ${ }^{7-13)}$ In addition, the revised American Heart Association and American College of Cardiology guidelines released in 2014 recommend either a $\mathrm{B}$ or a $\mathrm{M}$ valve in patients between 60 and 70 years of age (Level of Evidence: $B$ ), a B valve in patients aged more than 70 years, and an $M$ valve in patients aged less than 60 years (Level of Evidence: B). ${ }^{14)}$ On the other hand, there have been some reports that did not support lowering the cutoff age for $\mathrm{B}$ valves to less than 65 years because of insufficient evidence. ${ }^{15-17)}$ Forcillo et al. found that the rate of freedom from reoperation for SVD of the CarpentierEdwards pericardial valve averaged $60 \%$ and $30 \%$ at 15 and 20 years after surgery, respectively, in patients younger than 60 years of age compared with $90 \%$ at 15 years after surgery in patients 60 to 70 years of age and $99 \%$ at 10 years after surgery in patients older than 70 years of age. ${ }^{18)}$ Aupart et al. reported that actuarial freedom from SVD at 18 years varied from $99 \%$ in patients aged 70 years to $77 \%$ in those aged 60 to 70 years, and $45 \%$ in patients younger than 60 years and patient age was the only factor to influence durability. ${ }^{19)}$ Hanania suggested that $M$ valves should be recommended to all the patients aged less than 70 years because of their greater life expectancy and risks associated with reoperation. ${ }^{20)}$ Concerning the risk of reoperation, Potter et al. demonstrated that the risk of the reoperation is similar to that in primary AVR patients with a mean age of 64 years. ${ }^{21)}$ van Geldorp et al. reported that lifetime risk of reoperation was $25 \%$ with a $\mathrm{B}$ valve versus $3 \%$ with a $\mathrm{M}$ valve for patients aged 60 years. ${ }^{12}$

In 2013, Japanese women and men aged 60 years can expect to live another 28.5 and 22.8 years; at 65 years, they can expect to live another 19.1 and 24.0 years; and at 70 years, they can expect to live another and 19.6 and 15.3 years, respectively. Thus, patients aged between 65 and 70 years who undergo AVR can expect to outlive their bioprostheses, especially female patients, and there is a high probability that they will have to undergo reoperation as octogenarians. In this study, most staff surgeons in our facility followed the current Japanese guidelines and implanted $M$ valves in patients 60 to 65 years of age during the study period despite the low risk of reoperation. ${ }^{21)}$ Survival and freedom from reoperation were comparable between the two groups after propensity score matching preoperative characteristics. The most important concern when choosing valves in patients 60 to 70 years of age is not the risk of reoperation but the possibility of reoperation, which the patient will receive in his or her 80s. With this in mind, most patients in our facility tended to avoid $B$ valves despite the fact that $\mathrm{B}$ valves eliminate the risks of lifelong anticoagulation. Elderly high-risk patients with a degenerative bioprosthesis may be suitable for valve-in-valve implantation, ${ }^{22-24)}$ but most of the elderly Japanese patients with the implanted small valves (19 mm: 40\%, $21 \mathrm{~mm}: 34 \%$ ) may be poor candidates for valve-in-valve procedure with the current available technology. ${ }^{22,23)}$

Arom et al. reported a hemorrhage rate of only $0.48 \%$ per year and a thromboembolic rate of $0.8 \%$ per year with a target INR of 1.8 to $2.5 .^{25)}$ Torella et al. reported that a low INR range of 1.5 to 2.5 resulted in a similar rate of thromboembolic events and a significant reduction in bleeding complications compared with the standard range INR of 2.0 to 3.0. ${ }^{26)}$ Stassano et al. did not find any difference in the rate of bleeding between $\mathrm{M}$ and B valves with a target INR of 2.0 to 2.5. ${ }^{4)}$ In the VA trial, Hammermeister et al. suggested that a lower level of anticoagulation expected lower bleeding rate and $M$ valves would be beneficial in patients aged less than 65 years. ${ }^{3)}$ In Japan, the target INR is lower than that of most Western countries. ${ }^{24,25}$ ) The INR was maintained between 1.5 and 2.6 in this study and the rate of bleeding or thromboembolism showed less frequency. ${ }^{27)}$ The results underscored the efficacy of the anticoagulation regimen and call into question the belief that bioprostheses are associated with a lower embolic rate.

The main limitation of this study is that it was not a randomized but retrospective comparison between $\mathrm{B}$ and the $M$ valves. The relatively small size of the cohort and the use of multiple types of prosthetic valves in a single institution also potentially confounded the results. It remains unclear whether the durability and function differ among the different types of $\mathrm{B}$ and $\mathrm{M}$ valves used in this study. To answer these questions, prospective randomized 
trials are needed. Nevertheless, the present valve choices under the current Japanese guidelines reflected the physician's real thinking for small Japanese patients living in a rapidly aging and long-living society.

In summary, the current strategy of aortic valve choice based on the Japanese guidelines that recommend a $B$ valve for patients more than 65 years of age has provided excellent long-term results so far. But both physicians and patients must understand that implanting $B$ valves in patients around the criteria age increases the chance of that patient having to undergo reoperation in their 80s, and that this risk might outweigh the risks of chronic anticoagulation (especially when these risks have been shown to be lower in Japan than elsewhere). Moreover, over $70 \%$ of B valves were small valves (label size $\leq 21 \mathrm{~mm}$ ) and those valves may not be amenable to the transcatheter valve-in-valve procedure with the current available technology. We believe that the criteria age of 65 years is suitable and should not be lowered to take into account the increasing longevity of the Japanese population.

\section{Disclosure Statement}

The authors have no funding, no financial relationships, and no conflicts of interests.

\section{References}

1) Akins CW, Miller DC, Turina MI, et al. Guidelines for reporting mortality and morbidity after cardiac valve interventions. J Thorac Cardiovasc Surg 2008; 135: 732-8.

2) Oxenham H, Bloomfield P, Wheatley DJ, et al. Twenty year comparison of a Bjork-Shiley mechanical heart valve with porcine bioprostheses. Heart 2003; 89: 715-21.

3) Hammermeister K, Sethi GK, Henderson WG, et al. Outcomes 15 years after valve replacement with a mechanical versus a bioprosthetic valve: final report of the Veterans Affairs randomized trial. J Am Coll Cardiol 2000; 36: 1152-8.

4) Stassano P, Di Tommaso L, Monaco M, et al. Aortic valve replacement: a prospective randomized evaluation of mechanical versus biological valves in patients ages 55 to 70 years. J Am Coll Cardiol 2009; 54: 1862-8.

5) Wheatley DJ. The 'threshold age' in choosing biological versus mechanical prostheses in western countries. J Heart Valve Dis 2004; 13: S91-4.

6) Committee for Scientific Affairs, The Japanese Association for Thoracic Surgery, Masuda M, et al.
Thoracic and cardiovascular surgery in Japan during 2013: Annual report by The Japanese Association for Thoracic Surgery. Gen Thorac Cardiovasc Surg 2015; 63: 670-701.

7) Prasongsukarn K, Jamieson WR, Lichtenstein SV. Performance of bioprostheses and mechanical prostheses in age group 61-70 years. J Heart Valve Dis 2005; 14: 501-8, 510-1; discussion 509.

8) Ruggieri VG, Flecher E, Anselmi A, et al. Long-term results of the carpentier-edwards supraannular aortic valve prosthesis. Ann Thorac Surg 2012; 94: 1191-7.

9) Nishida T, Sonoda H, Oishi Y, et al. Long-term results of aortic valve replacement with mechanical prosthesis or carpentier-edwards perimount bioprosthesis in Japanese patients according to age. Circ J 2014; 78: 2688-95.

10) Stoica S, Goldsmith K, Demiris N, et al. Microsimulation and clinical outcomes analysis support a lower age threshold for use of biological valves. Heart 2010; 96: $1730-6$.

11) Chiang YP, Chikwe J, Moskowitz AJ, et al. Survival and long-term outcomes following bioprosthetic vs mechanical aortic valve replacement in patients aged 50 to 69 years. JAMA $2014 ; \mathbf{3 1 2}$ : 1323-9.

12) van Geldorp MW, Eric Jamieson WR, Kappetein AP, et al. Patient outcome after aortic valve replacement with a mechanical or biological prosthesis: weighing lifetime anticoagulant-related event risk against reoperation risk. J Thorac Cardiovasc Surg 2009; 137: 881-6, 886e1-5.

13) Dagenais F, Cartier P, Voisine $P$, et al. Which biologic valve should we select for the 45- to 65-year-old age group requiring aortic valve replacement? J Thorac Cardiovasc Surg 2005; 129: 1041-9.

14) Amsterdam EA, Wenger NK, Brindis RG, et al. 2014 AHA/ACC guideline for the management of patients with non-ST-elevation acute coronary syndromes: executive summary: a report of the American College of Cardiology/American Heart Association Task Force on Practice Guidelines. Circulation 2014; 130: 2354-94.

15) Kulik A, Bedard P, Lam BK, et al. Mechanical versus bioprosthetic valve replacement in middle-aged patients. Eur J Cardiothorac Surg 2006; 30: 485-91.

16) Brown ML, Schaff HV, Lahr BD, et al. Aortic valve replacement in patients aged 50 to 70 years: improved outcome with mechanical versus biologic prostheses. J Thorac Cardiovasc Surg 2008; 135: 878-84; discussion 884.

17) Roumieh M, Ius F, Tudorache I, et al. Comparison between biological and mechanical aortic valve prostheses in middle-aged patients matched through propensity score analysis: long-term results. Eur J Cardiothorac Surg 2015; 48: 129-36.

18) Forcillo J, Pellerin M, Perrault LP, et al. CarpentierEdwards pericardial valve in the aortic position: 25-years experience. Ann Thorac Surg 2013; 96: 486-93. 
19) Aupart MR, Mirza A, Meurisse YA, et al. Perimount pericardial bioprosthesis for aortic calcified stenosis: 18-year experience with 1133 patients. J Heart Valve Dis 2006; 15: 768-75; discussion 775-6.

20) Hanania G. Which heart valve prosthesis for patients aged between 60 and 70 years? Heart 2003; 89: 481-2.

21) Potter DD, Sundt TM, Zehr KJ, et al. Operative risk of reoperative aortic valve replacement. J Thorac Cardiovasc Surg 2005; 129: 94-103.

22) Azadani AN, Jaussaud N, Matthews PB, et al. Aortic valve-in-valve implantation: impact of transcatheterbioprosthesis size mismatch. J Heart Valve Dis 2009; 18: $367-73$.

23) Dvir D, Webb JG, Bleiziffer S, et al. Transcatheter aortic valve implantation in failed bioprosthetic surgical valves. JAMA 2014; 312: 162-70.
24) Ferrari E. Transapical aortic 'valve-in-valve' procedure for degenerated stented bioprosthesis. Eur J Cardiothorac Surg 2012; 41: 485-90.

25) Kaneko T, Vassileva CM, Englum B, et al. Contemporary outcomes of repeat aortic valve replacement: a benchmark for transcatheter valve-in-valve procedures. Ann Thorac Surg 2015; 100: 1298-304; discussion 1304.

26) Arom KV, Emery RW, Nicoloff DM, et al. Anticoagulant related complications in elderly patients with St. Jude mechanical valve prostheses. J Heart Valve Dis 1996; 5: 505-10.

27) Torella M, Torella D, Chiodini P, et al. LOWERing the INtensity of oral anticoaGulant Therapy in patients with bileaflet mechanical aortic valve replacement: results from the "LOWERING-IT" Trial. Am Heart J 2010; 160: 171-8. 